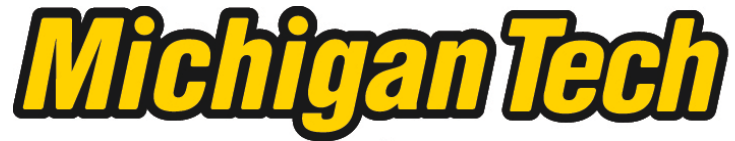 \\ Michigan Technological University Create the Future Digital Commons @ Michigan Tech
}

Dissertations, Master's Theses and Master's Reports - Open

Dissertations, Master's Theses and Master's

Reports

2011

\section{Enumeration of inequivalent cycle decompositions}

William J. Laffin

Michigan Technological University

Follow this and additional works at: https://digitalcommons.mtu.edu/etds

Part of the Mathematics Commons

Copyright 2011 William J. Laffin

\section{Recommended Citation}

Laffin, William J., "Enumeration of inequivalent cycle decompositions", Master's report, Michigan Technological University, 2011.

https://doi.org/10.37099/mtu.dc.etds/548

Follow this and additional works at: https://digitalcommons.mtu.edu/etds

Part of the Mathematics Commons 


\section{ENUMERATION OF INEQUIVALENT CYCLE DECOMPOSITIONS}

By

WILLIAM J. LAFFIN

\section{A REPORT}

Submitted in partial fulfillment of the requirements for the degree of MASTER OF SCIENCE

(Mathematical Sciences)

\section{MICHIGAN TECHNOLOGICAL UNIVERSITY}

2011

Copyright 2011 William J. Laffin 

This report, "Enumeration of Inequivalent Cycle Decompositions," is hereby approved in partial fulfillment of the requirements for the degree of MASTER OF SCIENCE IN MATHEMATICAL SCIENCES.

Department of Mathematical Sciences:

Signatures:

Thesis Advisor

Donald L. Kreher, Ph.D.

Committee Member

Phillip Merkey, Ph.D.

Committee Member

Charles Wallace, Ph.D.

Department Chair

Mark S. Gockenbach, Ph.D.

Date 


\section{Contents}

$\begin{array}{ll}\text { Abstract } & 7\end{array}$

1 Preliminary Theory and History 9

1.1 Graphs and Decompositions $\ldots \ldots \ldots \ldots \ldots$

2 Enumeration of Inequivalent Cycle Decompositions 13

2.1 Defining cycle decomposition arrays $\ldots \ldots \ldots \ldots \ldots$

2.2 How backtracking is used. . . . . . . . . . . . . . . 15

2.2.1 Remark on "current cycle" or "previous cycle" . . . . . . . . . . . . 15

2.3 Lexicographic Feasibility . . . . . . . . . . . . . . . . . . . . 15

2.4 Necessary and sufficient conditions for cycle decomposition arrays. . . . . . . . 17

2.5 Algorithm Development . . . . . . . . . . . . . . . . . . . 19

2.5.1 Backtrack search example 1: splitting $K_{7}$ into 3-cycles . . . . . . . . . 21

2.5.2 Backtrack search example 2: splitting $K_{5}$ into 5-cycles . . . . . . . . . 22

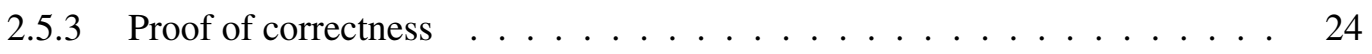

2.5 .4 Note on running time $\ldots \ldots \ldots \ldots \ldots$

2.6 Results and Future Projects . . . . . . . . . . . . . . . . . . . . 26

$\begin{array}{ll}\text { Bibliography } & 27\end{array}$

$\begin{array}{ll}\text { Appendix: Source code } & 28\end{array}$ 


\section{Abstract}

A k-cycle decomposition of order $\mathrm{n}$ is a partition of the edges of the complete graph on $\mathrm{n}$ vertices into k-cycles. In this report a backtracking algorithm is developed to count the number of inequivalent k-cycle decompositions of order $n$. 


\section{Chapter 1}

\section{Preliminary Theory and History}

In this chapter we discuss the relevant background with a short survey of results on cycle enumeration.

\subsection{Graphs and Decompositions}

A graph $X$ is a pair $(V(X), E(X))$, or simply $(V, E)$ such that $V$ is a set of points and $E$ is a multi-set of unordered pairs of points from $V$. The set $V$ is referred to as the vertex set while the set $E$ is referred to as the edge set. A simple graph is one such that $V$ is a finite set and $E$ is a set without repetition. The order of a graph is given by $|V(X)|$ while the size of a graph is given by $|E(X)|$. The degree of a vertex $x \in V(X)$ is the size of the set $\{e \in E(X): x \in e\}$. For example the graph $Y$ in Figure 1.1 has

$$
V(Y)=\{0,1,2,3,4,5\}
$$

and

$$
E(Y)=\{\{0,3\},\{0,4\},\{3,4\},\{1,3\},\{1,5\},\{3,5\},\{2,4\},\{2,5\},\{4,5\}\}
$$

The size of $Y$ is 9, while the order of $Y$ is 6 .

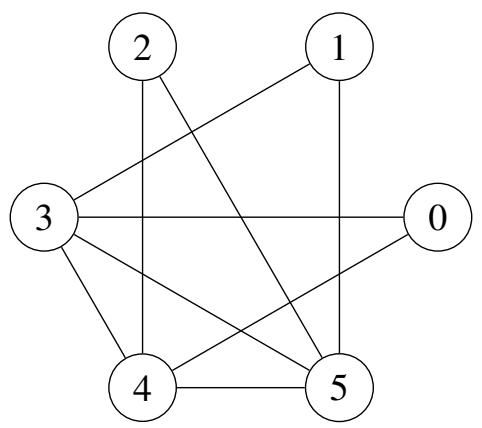

Figure 1.1: The graph $Y$ 


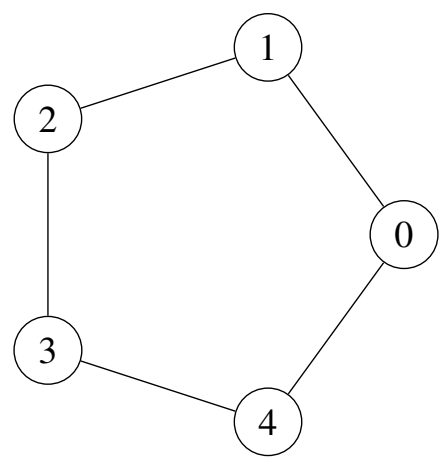

$\begin{array}{llllll}\text { Flips: } & (1,4)(2,3), & (2,0)(3,4), & (3,1)(4,0), & (4,2)(0,1), & (0,3)(1,2) . \\ \text { Rotations: } & (0)(1)(2)(3)(4), & (0,1,2,3,4), & (0,2,4,1,3), & (0,3,1,4,2), & (0,4,3,2,1) .\end{array}$

Figure 1.2: The automorphism group of $C_{5}$ is $D_{2 \cdot 5}$

The graph of all possible edges $K_{n}$ is called the complete graph. A subgraph $Y$ of a graph $X$ is a graph such that $V(Y) \subseteq V(X)$ and $E(Y) \subseteq E(X)$. For example, a subgraph $y$ of $Y$ in Figure 1.1 is given by

$$
V(y)=\{3,4,5\}
$$

and

$$
E(y)=\{\{3,4\},\{4,5\},\{3,5\}\} .
$$

Unless stated otherwise, in this report all graphs are given the vertex set $\{0,1, \ldots, n-1\}$. A walk on a graph $X$ is an array $A=\left(a_{0}, a_{1}, \ldots, a_{l-1}\right)$ of vertices such that $\left\{a_{i}, a_{i+1}\right\} \in E(X)$. A path on a graph $X$ is a walk such that no two vertices are the same. A cycle is a subgraph $C_{k}$ of a graph $X$ such that the vertices $V\left(C_{k}\right)$ can be arranged $c_{0}, c_{1}, \ldots, c_{k-1}$ such that

$$
E\left(C_{k}\right)=\left\{\left\{c_{i}, c_{i+1} \bmod k\right\}: 0 \leq i<k\right\} .
$$

We define $k$ the length of a cycle to be the number of vertices. For example, $C_{4}$ can be seen in graph $Y$ in Figure 1.1 by the array $1,3,4,5$.

An automorphism $\rho$ of a graph $X$ is a bijection on $V(X)$ such that

$$
\{\{\rho(x), \rho(y)\}:\{x, y\} \in E(X)\}=E(X)
$$

The permutation $1 \rightarrow 1,4 \rightarrow 4,3 \rightarrow 5,5 \rightarrow 3,0 \rightarrow 2,2 \rightarrow 0$ is an automorphism of $Y$ from Figure 1.1. Two graphs $X_{1}$ and $X_{2}$ are isomorphic if there is a bijection $\rho: V\left(X_{1}\right) \rightarrow V\left(X_{2}\right)$ such that

$$
\begin{aligned}
\left\{\rho(x): x \in V\left(X_{1}\right)\right\} & =V\left(X_{2}\right) \\
\left\{\{\rho(x), \rho(y)\}:\{x, y\} \in E\left(X_{1}\right)\right\} & =E\left(X_{2}\right)
\end{aligned}
$$

The automorphism group of a graph is the subgroup $\operatorname{Aut}(X)$ of $S_{V}$ that consists of all the automorphisms of $X$. For example, the dihedral group $D_{2 n}$ is the group of all automorphisms of the cycle on $n$ vertices. The size of this group is $2 n$ and consists of "flips" and "rotations". In Figure $1.2 D_{2.5}$ is shown in permutation notation, where $(a, b, c)$ means $a \rightarrow b \rightarrow c \rightarrow a$. 


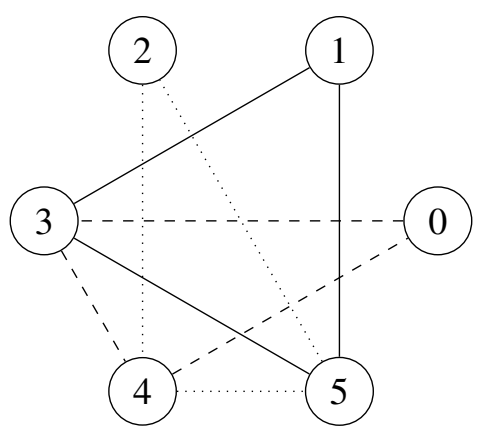

Figure 1.3: A 3-cycle decomposition of the graph $Y$ in Figure 1.1.

A decomposition of a graph $X$ into isomorphic copies of a subgraph $Y$ is a partition $\mathscr{D}=\left\{d_{1}, \ldots, d_{k}\right\}$ of $E(X)$ into disjoint sets such that each graph defined by $\left(\bigcup d_{i}, d_{i}\right)$ is isomorphic to $Y$. $\bigcup d_{i}$ here refers to the union of all edges in $d_{i}$. A $k$-cycle decomposition of a graph $X$ is an edge decomposition of $X$ into $k$-cycles. A 3-cycle decomposition of the graph $Y$ from Figure 1.1 is provided in Figure 1.3. Two $k$-cycle decompositions $\mathscr{D}_{1}, \mathscr{D}_{2}$ of a graph $X$ are said to be isomorphic if there is a $\rho \in \operatorname{Aut}(X)$ such that $d_{i} \in \mathscr{D}_{1}$ if and only if $\rho\left(d_{i}\right) \in \mathscr{D}_{2}$. An automorphism of a $k$-cycle decomposition is an isomorphism between a decomposition and itself. A cyclic $k$-cycle decomposition is one which $V(X)=\mathbb{Z}_{n}$ and $\rho(v)=v+1 \bmod n$ is an automorphism. We specify a $k$-cycle decomposition as a sequence of vertices partitioned into $k$-cycles. For example the 3-cycle decomposition of the graph $Y$ displayed in Figure 1.3 can be specified as

$$
\begin{aligned}
A & =(2,4,5)(1,3,5)(3,4,0) \\
\text { or } & =(0,3,4)(1,3,5)(2,4,5)
\end{aligned}
$$

Two $k$-cycle decompositions are equivalent if they consist of the same set of cycles, otherwise they are inequivalent. (In Chapter 2, for ease of exposition we often drop the inner parentheses and just write the sequence of vertices $\left.A=\left(a_{0}, a_{1}, \ldots, a_{m-1}\right)\right)$.

The $k$-cycle decomposition problem is finding $k$-cycle decompositions. Cycle Decomposition problems in general are $N P$-complete [5, 11]. A recent survey on cycle decompositions is [3].

For the purpose of enumeration we define 3 quantities, all on vertex set $\{0,1, \ldots, n-1\}$ :

- $\mathscr{M}(n, k)$ is the number of inequivalent $k$-cycle decompositions of $K_{n}$.

- $\mathscr{N}(n, k)$ the number of pairwise non-isomorphic $k$-cycle decompositions of $K_{n}$.

- $\mathscr{C}(n, k)$ the number of pairwise non-isomorphic cyclic $k$-cycle decompositions of $K_{n}$.

Table 1.1 summarizes known values of $\mathscr{M}(n, k), \mathscr{N}(n, k)$, and $\mathscr{C}(n, k)$.

Problem: What is the number of inequivalent $k$-cycle decompositions of the complete graph of $K_{n}$ ? What is $\mathscr{M}(n, k)$ ? 
Table 1.1: Enumeration Results.

\begin{tabular}{|llll|ll|ll|}
\hline$n$ & $k$ & $\mathscr{C}(n, k)$ & ref & $\mathscr{N}(n, k)$ & ref & $\mathscr{M}(n, k)$ & ref \\
\hline 5 & 5 & $?$ & - & $?$ & - & 6 & {$[10]$} \\
\hline 7 & 3 & 1 & {$[1]$} & 1 & - & 30 & - \\
7 & 7 & $?$ & - & $?$ & - & 960 & {$[10]$} \\
\hline 9 & 3 & 0 & {$[7]$} & 1 & - & 840 & - \\
9 & 4 & 1 & {$[1]$} & $?$ & - & $1,643,040$ & New \\
9 & 6 & $?$ & - & $?$ & - & $222,243,840$ & $N e w$ \\
9 & 9 & $?$ & - & 122 & {$[4]$} & $40,037,760$ & {$[10]$} \\
\hline 11 & 5 & 4 & {$[1]$} & $?$ & - & $?$ & - \\
11 & 11 & $?$ & - & $?$ & - & $?$ & - \\
\hline 13 & 3 & 1 & {$[7]$} & 2 & {$[6]$} & $1,197,504,000$ & {$[6]$} \\
13 & 6 & 16 & {$[1]$} & $?$ & - & $?$ & - \\
13 & 13 & $?$ & - & $?$ & - & $?$ & - \\
\hline 15 & 3 & 2 & - & 80 & {$[6]$} & $60,281,712,691,200$ & {$[6]$} \\
15 & 5 & $?$ & - & $?$ & - & $?$ & - \\
15 & 7 & 168 & {$[1]$} & $?$ & - & $?$ & - \\
15 & 15 & $?$ & - & $?$ & - & $?$ & - \\
\hline 17 & 4 & $?$ & - & $?$ & - & $?$ & - \\
17 & 8 & 696 & {$[1]$} & $?$ & - & $?$ & - \\
17 & 17 & $?$ & - & $?$ & - & $?$ & - \\
\hline 19 & 3 & 4 & {$[7]$} & $11,084,874,829$ & {$[6]$} & $* *$ & - \\
19 & 9 & 7,138 & {$[1]$} & $?$ & - & $?$ & - \\
19 & 19 & $?$ & - & $?$ & - & $?$ & {$[6]$} \\
\hline
\end{tabular}

$* *=1,348,410,350,618,155,344,199,680,000$

An integer $n$ is called $k$-admissible if $k \leq n$ and $k$ divides $\frac{n(n-1)}{2}$. 


\section{Chapter 2}

\section{Enumeration of Inequivalent Cycle Decompositions}

In this chapter we first provide lemmas needed to establish the correctness of the backtrack algorithm we used. For the remainder of this report, fix $n$ and $k$ and concentrate on the enumeration of $k$-cycle decompositions of $K_{n}$. The vertex set will be $V=\{0,1, \ldots, n-1\}$.

\subsection{Defining cycle decomposition arrays}

The following Lemma classifies all orbit representatives of the action of the symmetric group $S_{V}$ on the set of cycles. First we describe some characteristics of the orbit representative of cycles.

Lemma 1 All cycles of length $k$ have a representation as a array of length $k$. Furthermore, there is a unique lexicographically smallest array of vertices, $a_{0}, a_{1}, \ldots, a_{k-1}$, each pair $\left\{a_{i}, a_{i+1}\right\}$ (subscript addition taken $\bmod k$ ) is an edge in the cycle and

1. $a_{0}<a_{i}$ for all $1 \leq i<k$,

2. $a_{1}<a_{k-1}$

Proof. By definition, there exists at least one ordering. Because the automorphism group of a $k$ cycle is $D_{2 k}$ there are $2 k$ ways to order the vertices. Because there are rotations in $D_{2 k}$, we can cyclically shift the array as needed for condition 1 . Also because there are flips in $D_{2 k}$, we can fix the first element and flip the rest of the cycle as needed for condition 2.

Without loss let $a_{0}, a_{1}, \ldots a_{n-1}$ be an ordering such that $a_{0}<a_{i}$ for all $1 \leq i<k$. Because there are flips in $D_{2 n}, a_{0}, a_{n-1}, a_{n-2}, \ldots, a_{1}$ is a possible ordering of the vertices.

Because these two are flips of each other, part 2 picks the lexicographically smallest one, as $a_{k-1}$ is the reversed cycle's $a_{1}$. 
We refer to the above representation as a cycle array. Note that there are $\left(\begin{array}{l}n \\ 2\end{array}\right) / k$ cycles in a cycle decomposition.

Lemma 2 There are $\left(\begin{array}{l}n \\ k\end{array}\right) \frac{(k-1) !}{2} k$-cycles in the complete graph.

Proof. First we choose the vertex set. There are $\left(\begin{array}{l}n \\ k\end{array}\right)$ ways to do this. Second, we choose the ordering. By the orbit counting lemma, the number of orbits is equal to the size of the group $(k !)$ divided by the size of the automorphism group $2 k$.

Now that we have a cycle array, we can then create a unique representation for cycle decompositions.

Lemma 3 Let $D=\left\{d_{1}, \ldots, d_{\left(\begin{array}{c}n \\ 2\end{array}\right) / k}\right\}$, where $d_{i}, 1 \leq i \leq\left(\begin{array}{c}n \\ 2\end{array}\right) / k$, is a cycle, be a $k$-cycle decomposition of $K_{n}$. Then $D$ has a lexicographically smallest representation as an array of vertices $\left(a_{0}, a_{1}, \ldots, a_{\left(\begin{array}{c}n \\ 2\end{array}\right)-1}\right)$ made by concatenating, in lexicographic order, the cycle arrays of each $d_{i}$ where

1. Each vertex is represented $\frac{n-1}{2}$ times

2. $\left(a_{j}, a_{j+1}, \ldots, a_{j+k-1}\right)$, where $j=k * i$ for some integer $0 \leq i<\left(\begin{array}{c}n \\ 2\end{array}\right) / k$, is the ith lexicographically smallest cycle.

Proof. Each lexicographically smallest array representing a cycle decomposition is a lexicographical ordering of all cycles contributing to the decomposition. The order is the number of edges in the graph, $\left(\begin{array}{l}n \\ 2\end{array}\right)$. Note that each vertex in the complete graph has degree $(n-1)$. Also note that each vertex in a cycle has degree 2 . Thus it follows that each vertex appears $\frac{n-1}{2}$ times, and consequently the first $\frac{n-1}{2}$ cycles in the array start with 0 . As each cycle takes up exactly $k$ vertices, we know that the starting position of each cycle is $0 \bmod k$. The number of cycles at any position $j$ is $\lfloor j / k\rfloor$.

We refer to this smallest representation as a cycle decomposition array. We define two cycle decompositions to be inequivalent if their cycle decompositions arrays differ.

Alspach, Gavlas, Šajna settled that these necessary conditions are also sufficient.

Theorem 4 (Alspach-Gavlas 2001 [2], Šajna 2002 [9]) A $k$-cycle decomposition of $K_{n}$ exists if and only if $n$ is $k$-admissible.

We note some consequences of Theorem 4 relative to $\mathscr{M}(k, n)$. Because $\mathscr{M}(k, n)$ is the number of inequivalent cycle decompositions of $K_{n}$ into $k$ cycles, then if $n$ is not $k$-admissible $\mathscr{M}(k, n)=0$. Also if $n<k$, then $\mathscr{M}(k, n)=0$. In this report we are concerned with computing $\mathscr{M}(k, n)$ such that $n$ is $k$-admissible. 


\subsection{How backtracking is used.}

Our overall goal is to count the number of inequivalent cycle decompositions of $K_{n}$. Our method of enumeration relies on a backtracking search. We compute $\mathscr{M}(n, k)$ by recursively attempting to extend partial solutions lexicographically.

A partial solution of length $l$ to the $k$-cycle decomposition problem is an array $A=\left(a_{0}, a_{1}, \ldots, a_{l-1}\right)$ such that the $c=\lfloor l / k\rfloor$ cycles

$$
\left(a_{j k}, a_{j k+1}, \ldots, a_{j(k+1)-1}\right), j=0,1, \ldots, c-1
$$

and the path

$$
a_{c k}, a_{c k+1}, \ldots, a_{l-1}
$$

are all pairwise edge disjoint. Let $E(A)$ be the set of edges covered by the partial solution $A$. A partial solution is a complete solution if $|E(A)|=\left(\begin{array}{l}n \\ 2\end{array}\right)$. Let $\mathcal{A}$ be the set of all partial solutions to the $k$-cycle decomposition problem.

A partial solution $A=\left(a_{0}, a_{1}, \ldots, a_{l-1}\right) \in \mathcal{A}$ of length $l$ may be extended to a partial solution $\left(a_{0}, a_{1}, \ldots, a_{l-1}, x\right) \in \mathcal{A}$ of length $l+1$ if $x$ can be chosen such that $x \notin\left\{a_{c k}, a_{c k+1}, \ldots, a_{l-1}\right\}$ and

$$
\begin{array}{r}
\left\{a_{l-1}, x\right\} \notin E(A) \text { when } l \not \equiv 0,-1 \quad \bmod k \\
\left\{a_{l-1}, x\right\},\left\{a_{c k}, x\right\} \notin E(A) \text { when } l \equiv-1 \quad \bmod k
\end{array}
$$

where $c=\lfloor l / k\rfloor$.

In order to avoid duplication of effort in our backtrack search, we impose a total ordering " $\leq$ lex" on $\mathcal{A}$. If $A=\left(a_{0}, a_{1}, \ldots, a_{l_{1}-1}\right), B=\left(b_{0}, b_{1}, \ldots, b_{l_{2}-1}\right) \in \mathcal{A}$ when $a_{i}=b_{i}$ for $i=0,1, \ldots, j-1$, $j \leq \min \left\{l_{1}, l_{2}\right\}$, then $A \leq_{\text {lex }} B$ if $a_{j}<b_{j}$.

\subsubsection{Remark on "current cycle" or "previous cycle"}

In this report, when the terms current cycle or new cycle and previous cycle are used, there is position $i$ indexing an array $A \in \mathcal{A}$ that is the location of this cycle. More precisely, let $j=i \bmod k$, and $c=i-j$. Then current cycle and new cycle refer to the (possibly empty) subarray $a_{c}, \ldots, a_{i}$ and previous cycle refers to the (possibly empty) subarray $a_{c-k}, \ldots, a_{c-1}$. A prior cycle refers to any of the subarrays $a_{c-j * k}, \ldots, a_{c-(j-1) k-1}$ for $1 \leq j \leq\lfloor i / k\rfloor$.

\subsection{Lexicographic Feasibility}

In this section we describe a range of values that determine how a partial solution may be extended to a lexicographically larger partial solution. 
Lemma 1 and Lemma 3 describe necessary restrictions for the "profile" of each cycle in the cycle decomposition array. By Lemma 1, the first element of each cycle must be the smallest, and the second element must be smaller than the last. By Lemma 3 each cycle must, each in turn, be lexicographically larger than the previous. For each partial solution to be extended correctly, these Lemmas are checked at every step. Lemmas 5 and 6 show that these restrictions form ranges of values $\left\langle s_{i}, e_{i}\right\rangle$.

For example, consider a partial solution $A=(5)$. One cannot extend to $A=(5,3)$ because $3<5$, failing to pass the characterization of cycles set by Lemma 1 that $a_{0}<a_{1}$.

We begin by extending a partial solution to create the first cycle.

Lemma 5 Let $i<k$. Necessary conditions for lexicographically extending the partial solution

$$
A=\left(a_{0}, a_{1}, \ldots, a_{i-1}\right)
$$

to the partial solution $A=\left(a_{0}, a_{1}, \ldots, a_{i-1}, v\right)$ are that $v \in\left\{s_{i}, s_{i}+1, \ldots, e_{i}\right\}$ where

$$
\left(s_{i}, e_{i}\right)= \begin{cases}(0,0) & \text { if } i=0 \\ (1, n-2) & \text { if } i=1 \\ (1, n-1) & \text { if } 2 \leq i \leq k-2 \\ \left(a_{1}+1, n-1\right) & \text { if } i=k-1\end{cases}
$$

Proof. By Lemma 1 the first $\frac{n-1}{2}$ cycles must begin with 0 . By Lemma $1 a_{0}$ must be the smallest in the array. By Lemma $2 a_{k-1}>a_{1}$.

In the above proof, Lemma 3 imposes no conditions because $i<k$.

The (lexicographically) largest cycle array $(n-k, n-2, n-3, \ldots, n-k-1, n-1)$ is naturally composed of the largest set of elements. By Lemma 2, the smallest element is first $a_{0}=n-k$. Making $a_{1}$ the largest value then involves $a_{1}<a_{k-1}$, thus $a_{1}=n-2$ and $a_{k-1}=n-1$. The rest of the possible values are ordered in descending order. We say $z$ is in the range $[x, y)$ to mean $x \leq z<y$ and $z \in \mathbb{Z}$.

Lemma 6 Let $k \leq i<\left(\begin{array}{c}n \\ 2\end{array}\right)$, and assume necessary conditions hold for all $0 \leq j<i$. Let $i_{n}=i$ $\bmod k$. Necessary conditions for lexicographically extending the partial solution

$$
A=\left(a_{0}, a_{1}, \ldots, a_{i-1}\right)
$$

to the partial solution $A=\left(a_{0}, a_{1}, \ldots, a_{i-1}, v\right)$ are that $v \in\left\{s_{i}, s_{i}+1, \ldots, e_{i}\right\}$ where

$$
\left(s_{i}, e_{i}\right)= \begin{cases}(0,0) & \text { if } i_{n}=0 \text { and } i / k<(n-1) / 2 \\ \left(a_{i-k}, n-k-1\right) & \text { if } i_{n}=0 \text { and } i / k \geq(n-1) / 2 \\ \left(a_{i-k}, n-2\right) & \text { if } i_{n}=1 \text { and } a_{i-1}=a_{i-1-k} \\ \left(a_{i-1}, n-2\right) & \text { if } i_{n}=1 \text { and } a_{i-1} \neq a_{i-1-k} \\ \left(a_{i-i_{n}+1}, n-1\right) & \text { if } i_{n}=k-1 \\ \left(a_{i-i_{n}}+1, n-1\right) & \text { if } i_{n} \notin\{0,1, k-1\}\end{cases}
$$


Proof. Note that facts mentioned in the proof for Lemma 5 now apply to the current cycle, $a_{i-i_{n}}, \ldots, a_{i-1}$. We split the analysis into cases based on $i_{n}$.

Let $i_{n}=0, a_{i}$ needs to be 0 for the first $\frac{n-1}{2}$ cycles. After that, the cycle needs to be the same or greater (lexicographically) than the previous cycle, so $a_{i}$ in this case is always in the range $\left[a_{i-k}, n-k\right)$,where the upper bound $n-k$ comes from the fact that there needs to be $k-1$ elements in the array past this point.

Let $i_{n}=1, a_{i}$ in this case depends on the profile of the previous cycle. This new cycle needs to be lexicographically larger than the previous so if $a_{i-1}=a_{i-1-k}$ then the range is specified by $\left[a_{i-k}, n-1\right)$ where $n-1$ is not possible because then the cycle's end could not exist. Otherwise, the range is specified by $\left[a_{i-1}, n-1\right)$ because of Lemma 2 .

Let $i_{n}=k-1, a_{i}$ in this case depends on the first element in the cycle. The position of this vertex in the array is $i-i_{n}+1$.

Let $i_{n} \notin\{0,1, k-1\}, a_{i}$ needs to be bounded by the first element in the array.

\subsection{Necessary and sufficient conditions for cycle decomposition ar- rays.}

In this section we develop necessary and sufficient conditions for determining when an array $A$ is a partial cycle decomposition array.

Define $I: \mathcal{A} \rightarrow \mathbb{Z}_{\left(\begin{array}{c}n \\ 2\end{array}\right)}$ by $I(A)$ is the smallest position $i$ such that one of the following conditions does not hold. Lacking this, $I(A)$ is set to the length of the array.

(I.Lexicographic Condition) With $i<\left(\begin{array}{l}n \\ 2\end{array}\right), a_{i}$ is in the range specified by Lemma 5 and Lemma 6.

(I.Edge Condition) Let $i_{n}=i \bmod k$ and

$$
L_{i}=\left\{\begin{array}{ll}
\{\} & \text { if } i_{n}=0 \\
\left\{\left\{a_{i-1}, a_{i}\right\}\right\} & \text { if } 1 \leq i_{n}<k-1 \\
\left\{\left\{a_{i-1}, a_{i}\right\},\left\{a_{i-(k-1)}, a_{i}\right\}\right\} & \text { if } i_{n}=k-1
\end{array} .\right.
$$

Let $\mathscr{L}=\bigcup L_{j}$ where $0 \leq j \leq i-1$. Then $\mathscr{L} \cap L_{i}=\emptyset$.

(I.Vertex Condition) Let $i_{n}=i \bmod k$. Then $a_{i} \notin\left\{a_{i-i_{n}}, \ldots, a_{i-1}\right\}$.

If none of the above conditions fail, we set $I(A)=|A|$. 
Lemma 7 A array $D$ is an array of lexicographically feasible cycles if and only if conditions (I.Lexicographic Condition) and (I.Vertex Condition) of the above definition are satisfied.

Proof. First assume $D$ is an array of cycles. By Lemmas 5 and $6, s_{i} \leq a_{i} \leq e_{i}$ and thus $I(A)$ condition (I.Lexicographic Condition) passes always. For condition (I.Vertex Condition), assume otherwise. Then there exists some $j$ such that $I(A)=j$ as per (I.Vertex Condition). Thus there is a vertex repeated in one subarray. However, this is an array of cycles, contradiction.

Conversely, assume conditions (I.Vertex Condition) and (I.Lexicographic Condition) are never broken. Condition (I.Vertex Condition) passing directly shows that each of the arrays are cycles, as they are arrays of different vertices. Condition (I.Lexicographic Condition) passing then shows that each of these cycles pass conditions imposed by Lemma 2 and Lemma 4.

Theorem $8 I(A)=\left(\begin{array}{l}n \\ 2\end{array}\right)$ if and only if $A$ is a cycle decomposition.

Proof. First assume $I(A)=\left(\begin{array}{l}n \\ 2\end{array}\right)$. By Lemma 7, $I(A)=\left(\begin{array}{l}n \\ 2\end{array}\right)$ implies $A$ is an array of cycles. By (I.Edge Condition) we know that no edge is covered twice. Then $I(A)=\left(\begin{array}{l}n \\ 2\end{array}\right)$ now implies that $A$ is a cycle decomposition.

Conversely assume $A$ is a cycle decomposition. By Lemma 7, $I(A)$ was not decided by (I.Lexicographic Condition) or (I.Vertex Condition). A cycle decomposition implies no edges are covered twice, so thus part (I.Edge Condition) passes always. From these three passing, we know that $I(A)=\left(\begin{array}{l}n \\ 2\end{array}\right)$.

Define the set of partial cycle decomposition arrays, PCDAs to be $K$.

$$
K=\{A \in \mathcal{A}: I(A)=|A|\}
$$

is the set of arrays in $\mathcal{A}$ that, which upon extending with a correct vertices, could be cycle decompositions. Then our backtrack algorithm iterates through each element of $K$. 


\subsection{Algorithm Development}

In this section, we present algorithm FIND-CyCLES to compute $\mathscr{M}(n, k)$. We also present a proof of correctness along with rudimentary run time analysis.

The algorithm FIND-CyCLES first finds data for the current cycle. Then it searches for new cycles, but first calculates the range of vertices it needs to iterate over by calling CALCULATE-RANGE.

At the beginning, Lines 1-6, FIND-CyCLES initializes local variables. The variable $i_{k}$ refers to the position in the current cycle, $c$ refers to the position of the start of the current cycle, newcyc refers to the address of the start of the current cycle, and prevcyc is a pointer to the start of the previous cycle.

Then, the basic recursive exit check on Line 7 will add one to num-found if the array is large enough. Otherwise the program will continue to build arrays in Lines 8-16.

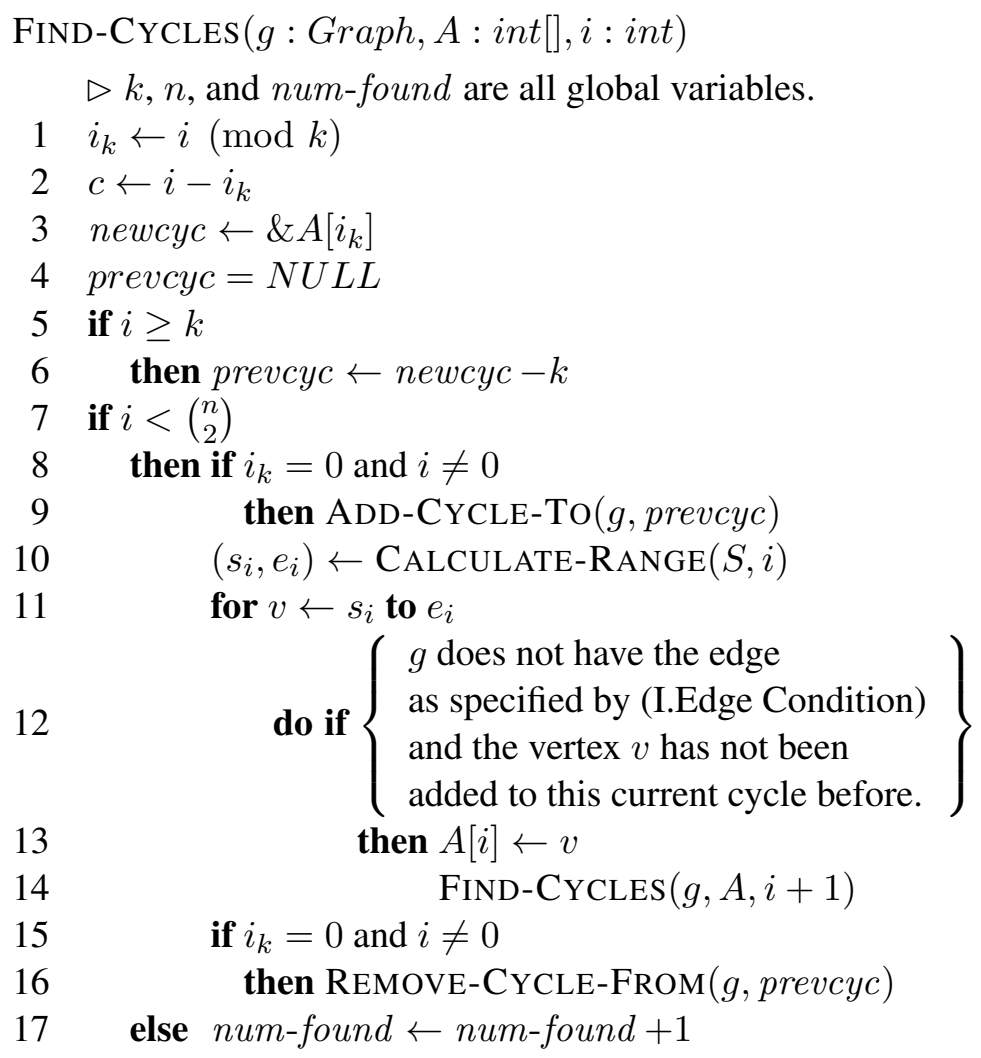




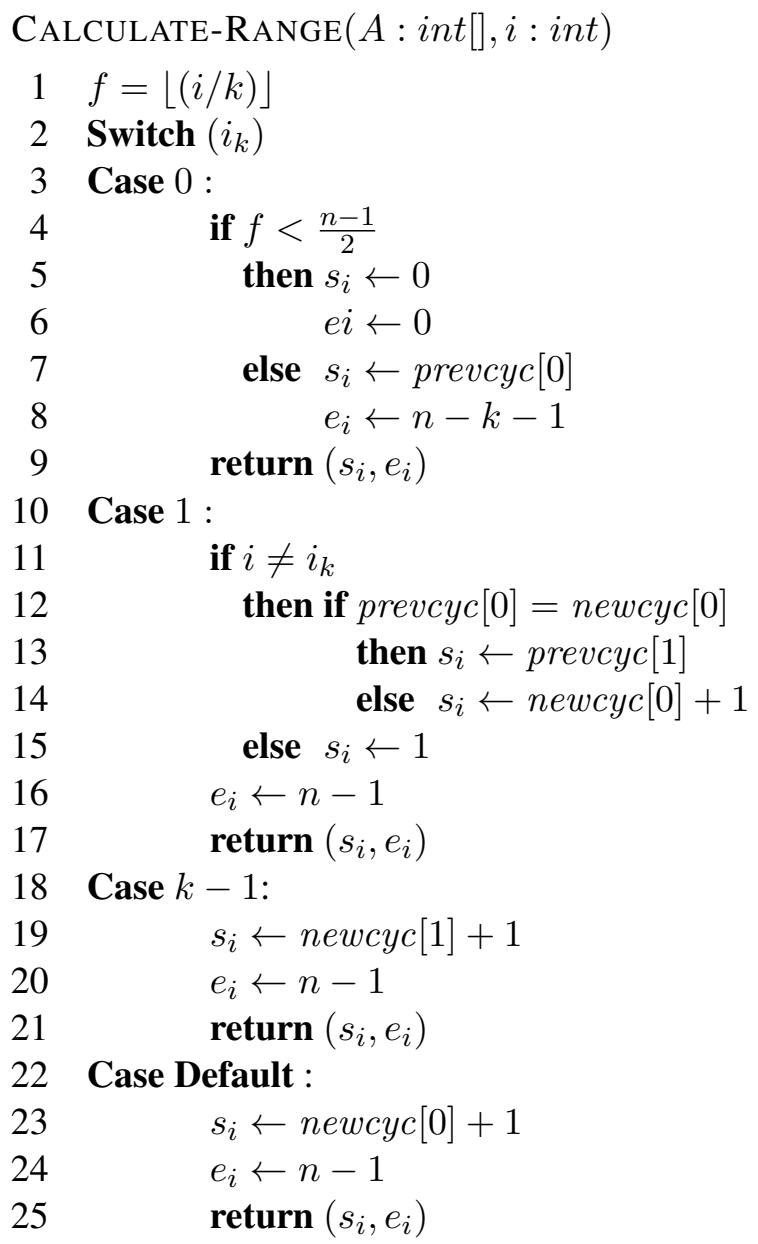

First this section, Lines 8-9 will add cycles to the graph of recording, kept by pointer $g$. The cycles will only be added if the previous call of FIND-CYCLES finished a cycle. Similarly, the end of this section, Lines 15-16, will remove the cycle, as this call is returning and will thus replace the last element in the previous cycle.

Sandwiched between these two checks, Lines 10-14, are where $I(S)$ is insured. The main point here is that FIND-CyCLES $(g, S, i+1)$ is called after extending the array only if $I(S)=i$. The range is calculated, so that (I.Lexicographic Condition) is satisfied, then the if statement on line 12 checks both (I.Vertex Condition) and (I.Edge Condition).

The algorithm CALCULATE-RANGE is strictly to return the ranges as specified by Lemmas 5 and 6 . 


\subsubsection{Backtrack search example 1: splitting $K_{7}$ into 3-cycles}

As described above, FIND-CyCLES computes $\mathscr{M}(n, k)$ through attempting to extend each possible partial solution. Consequently the backtrack search exhibited below yields a proof that

$$
(012)(034)(056)(135)(146)(236)(245)
$$

is the lexicographically smallest 3-cycle decomposition of $K_{7}$. New lines represent new indexes evaluated. Vertices in square brackets are not possible for reasons given as a short key corresponding to the list below.

edge already taken See (I.Edge Condition). If a vertex appears within square brackets proclaiming "[1 edge already taken]" then the edge(s) this vertex would have added to the graph were already covered.

in current cycle See (I.Vertex Condition). If a vertex fails and quickly pounces to the penalty box with the exclamation "[2 in current cycle]" it means that even though this vertex is in the range specified by Lemmas 5 and 6, this vertex has already appeared in the current cycle, so the cycle would close in on itself early if this vertex were to be added.

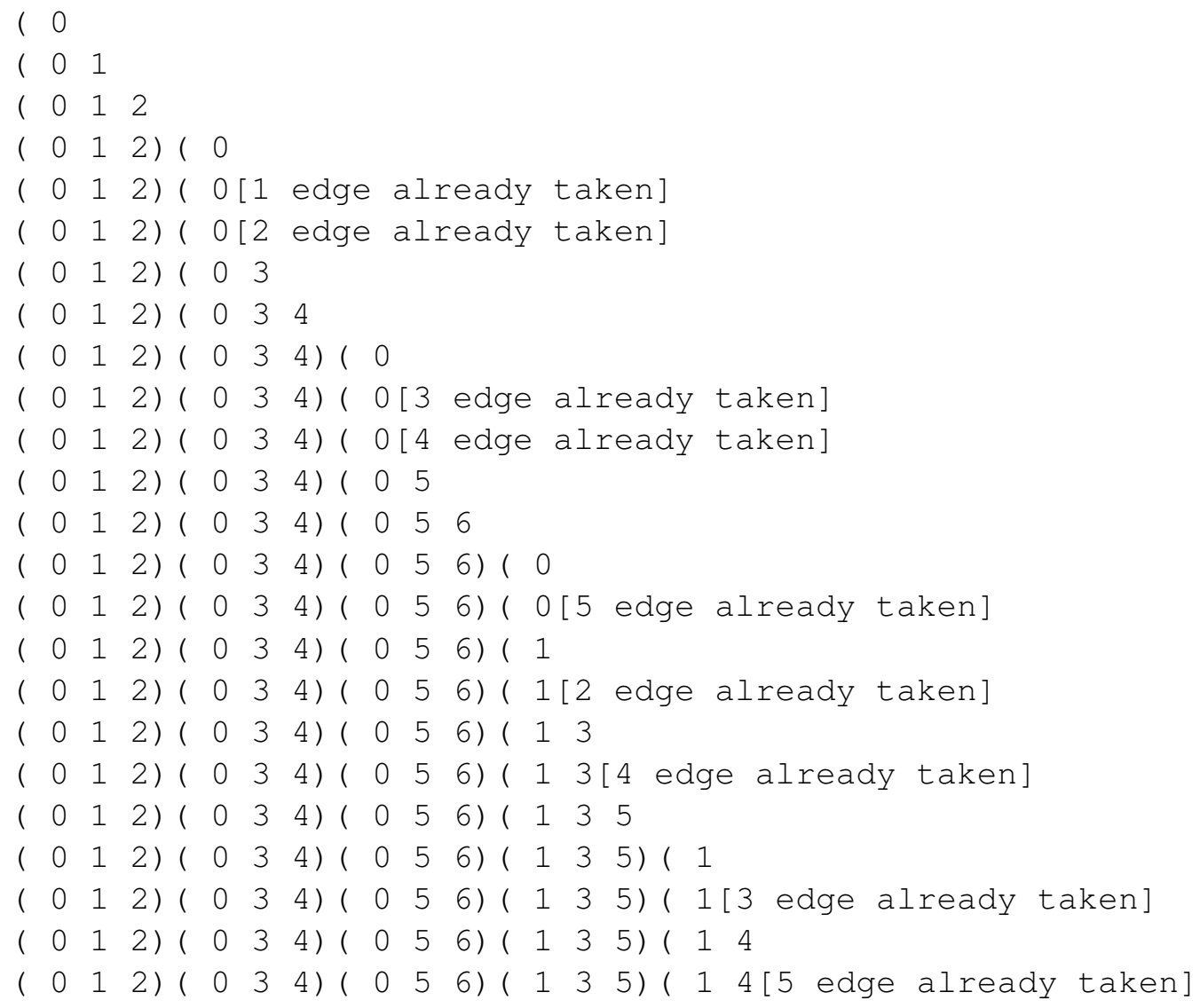




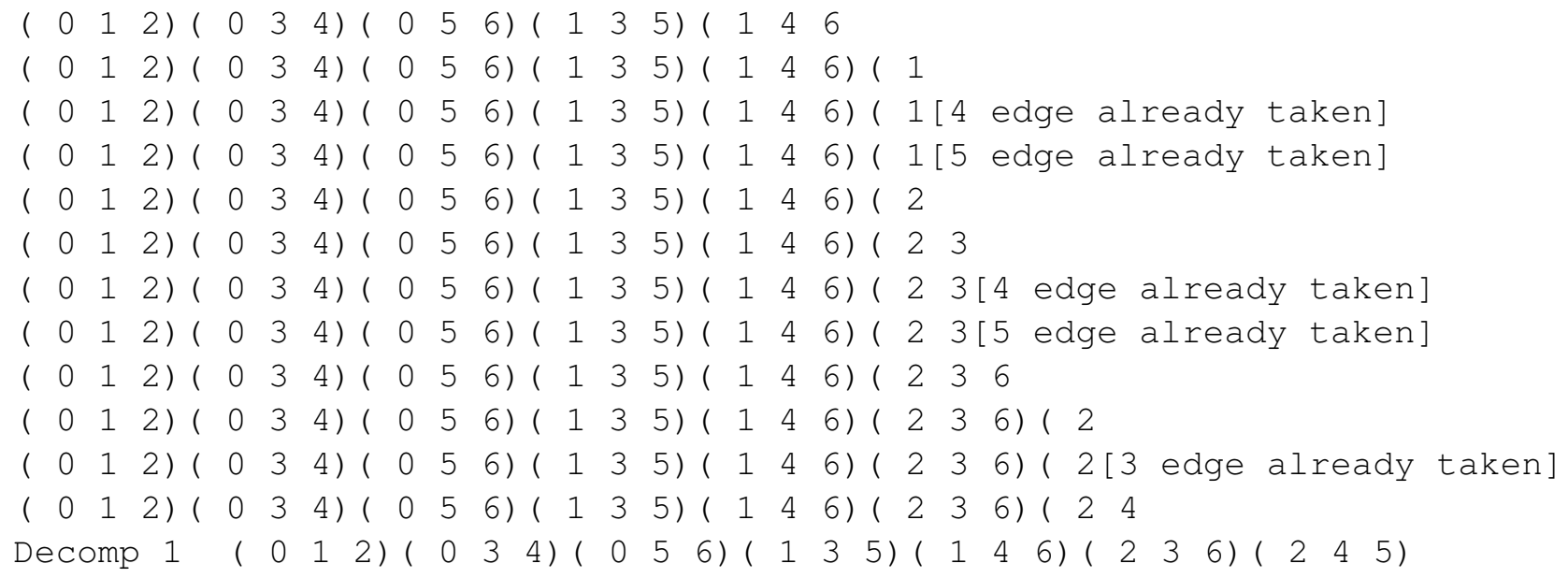

\subsubsection{Backtrack search example 2: splitting $K_{5}$ into 5-cycles}

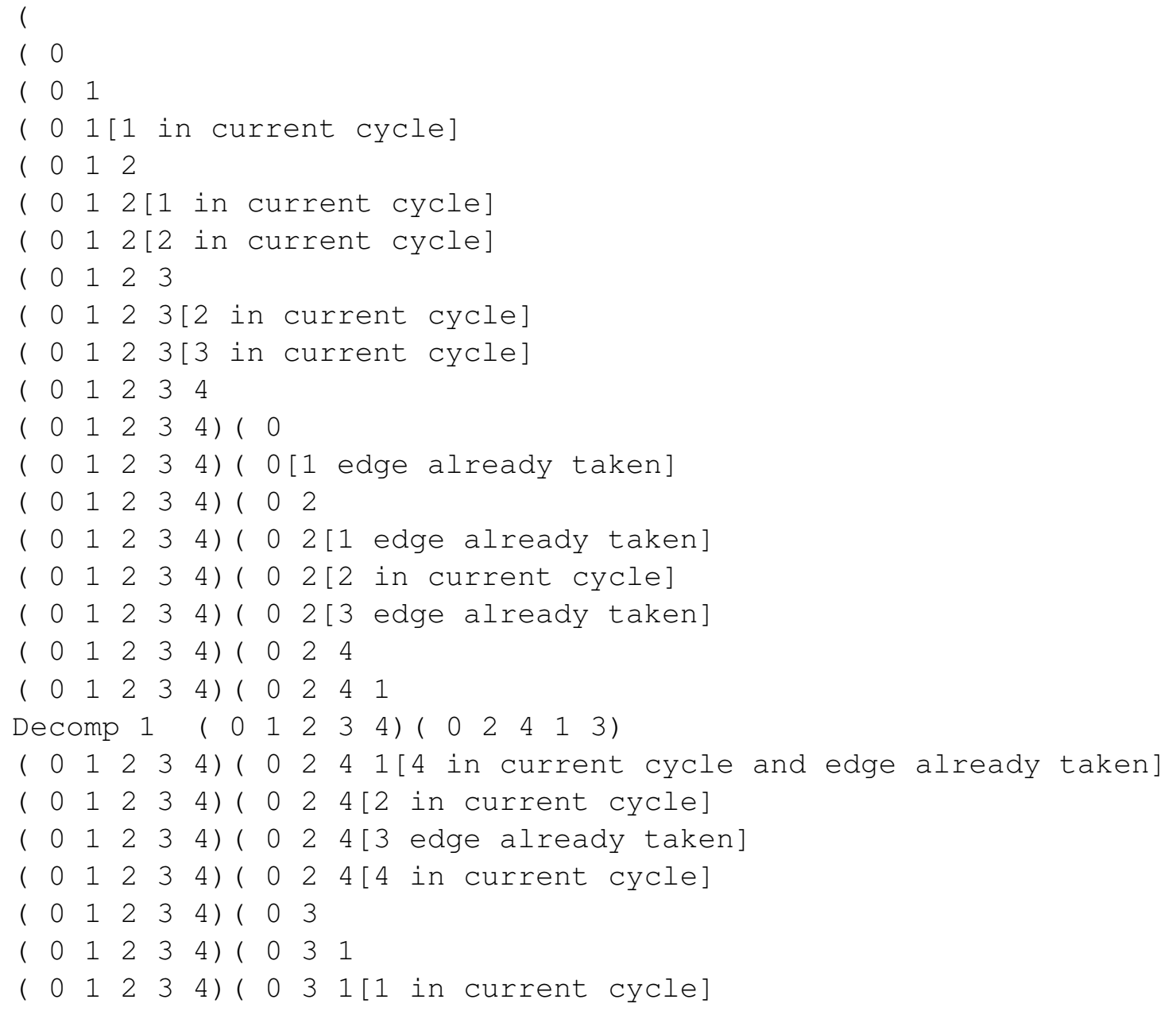




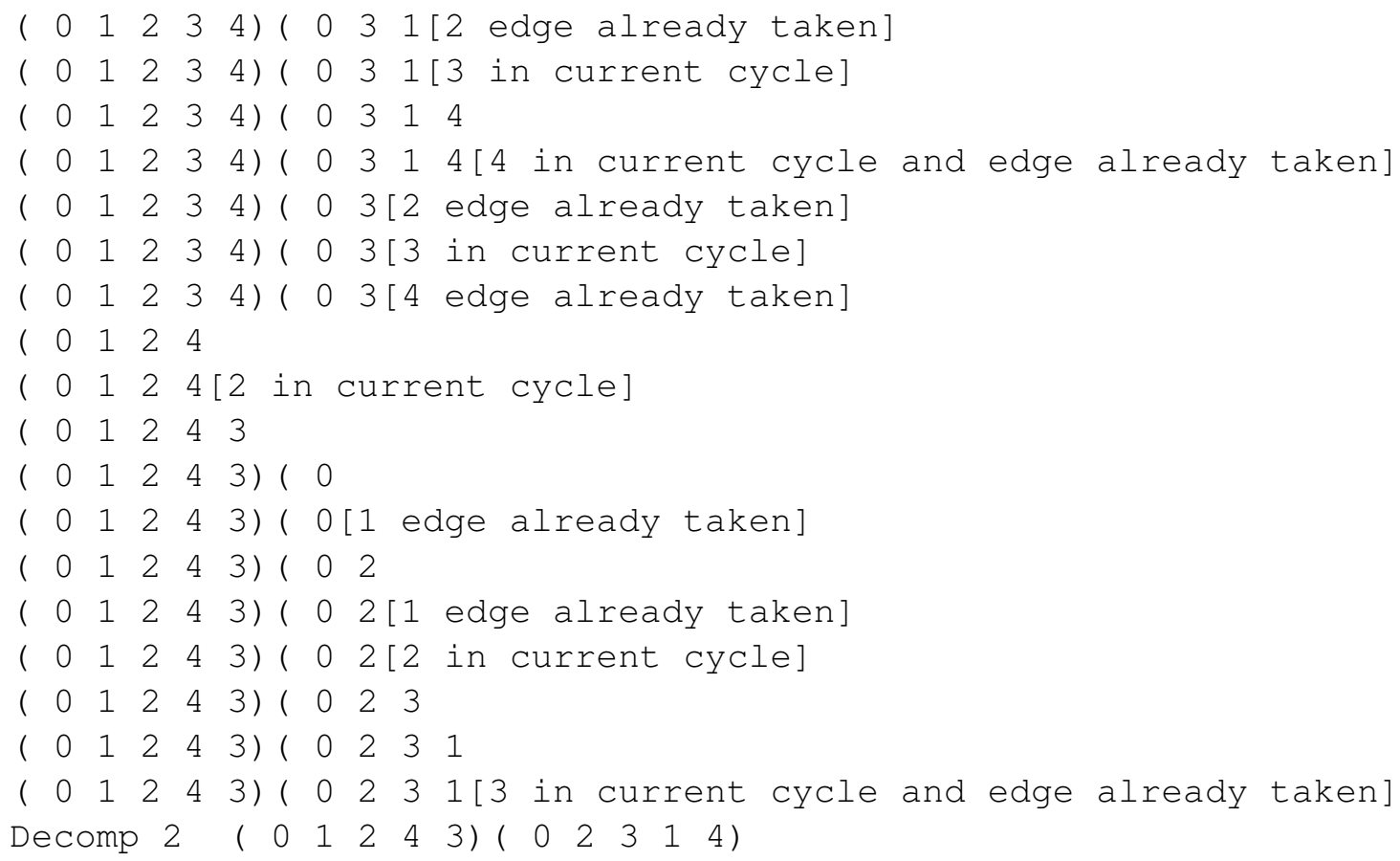




\subsubsection{Proof of correctness}

In this section we prove that FIND-CyCLES $(\emptyset, a, 0)$ correctly computes $\mathscr{M}(n, k)$, where $\emptyset$ denotes the empty graph. The proof is acomplished by estsblishing the following two items on the set

$$
\mathcal{I}=\{(k, n): n \text { is } k \text {-admissible. }\}
$$

of input parameters.

1. Show that FIND-CyCLES halts on parameters in $\mathcal{I}$.

2. Show that FIND-CYCLES gives the correct output.

We begin with two useful observations that can be easily deduced from the algorithm.

Observation 9 Let $A=\left(a_{0}, \ldots, a_{i-1}\right)$ be a partial solution. Then

CAlculate- $\operatorname{RAngE}(A, i)$ returns $\left(s_{i}, e_{i}\right)$ the start and end range as specified by Lemmas 5 and 6 .

Observation 10 The line 12 in FIND-CYCLES correctly checks if a vertex $v$ passes $I(A)$ edge check (I.Edge Condition) that $v$ passes $I(A)$ vertex check (I.Vertex Condition).

Lemma 11 shows that FIND-CYCLES halts for given input parameters.

Lemma 11 Let $(k, n) \in \mathcal{I}$ and let $A$ an array of size $\left(\begin{array}{l}n \\ 2\end{array}\right)$. Then $\operatorname{Find-CyCLES}(\emptyset, S, 0)$ halts.

Proof. Let Find-CyCLes $(X, A, i)$ be an arbitrary call in the call stack for the algorithm. Then line 11 insures that there is a finite branch factor, that FIND-CYCLES will be called a finite number of times.

Continuing, note that line 11 is only reached when $i<\left(\begin{array}{l}n \\ 2\end{array}\right)$, ensuring a finite depth. Thus there is a finite branching factor, and a finite depth factor, and thus the algorithm always returns.

The relation between line 14 and the function $I(A)$ is made clear in Lemma 12 .

Lemma 12 Let $j<\left(\begin{array}{c}n \\ 2\end{array}\right)$ and let $S$ be an array of size $\left(\begin{array}{c}n \\ 2\end{array}\right)$. Also let $A=S_{0}, \ldots, S_{j-1}$ such that $A$ is a PCDA and let $X$ be the graph defined as the union of all the cycles completed in $A$. Then line 14 of FIND-CyCLES $(X, S, j)$ is only called forming PCDAs with length $j$ in which $I\left(A_{\text {new }}\right)=\left|A_{\text {new }}\right|=j$.

Proof. From Observation 9 we know that the position added will not be outside the range defined by (I.Lexicographic Condition). From Observation 10 we know that an position added will pass the checks defined by (I.Edge Condition) or (I.Vertex Condition). Thus since $j<\left(\begin{array}{l}n \\ 2\end{array}\right), I\left(A_{\text {new }}\right)=$ $\left|A_{\text {new }}\right|=j$.

Thus we have shown that FIND-CYCLES will only be called with admissible values of $I(A)$. We now show how the set traversed is well ordered. 
Theorem 13 Let $S$ an array of size $\left(\begin{array}{l}n \\ 2\end{array}\right)$. Then, at the beginning of each call to FIND-CYCLES $(X, S, i)$, each admissible partial cycle system that is lexicographically smaller than $A=S_{0}, \ldots, S_{i-1}$ been visited already.

Proof. Assume otherwise, then there exists a lexicographically smaller partial cycle system array $L=\left(l_{i}\right)$ with an position of difference $j$ such that $a_{i}=l_{i}$ for $i<j$ and $a_{j}>_{\text {lex }} l_{j}$ that has not been visited by FIND-CYCLES. Call this subarray of equality $J$. However, since $A$ was built by Find-CyCLES then we can conclude there was a call Find-CyCLes $(g, J, j)$. During this call $j<\left(\begin{array}{l}n \\ 2\end{array}\right)$. From Observation 9 we know that for each admissible next vertex, FIND-CYCLES was called. However this contradicts our assumption that $L$ was not visited.

We conclude with the following theorem.

Theorem 14 Let $\emptyset$ be the empty graph and $S$ an array of size $\left(\begin{array}{l}n \\ 2\end{array}\right)$. Then the algorithm FIND-CyCLES $(\emptyset, S, 0)$ correctly computes $\mathscr{M}(n, k)$.

Proof. By Lemma 12, Find-CyCLes $(X, A, n c)$ will be called with $I(A)=\left(\begin{array}{l}n \\ 2\end{array}\right)$. Thus line 17 will be called and $A$ will be counted towards $\mathscr{M}(n, k)$. Thus every cycle decomposition array will be counted at least once. And by Theorem 13, every cycle decomposition array will be counted just once, and in lexicographic order.

\subsubsection{Note on running time}

We see that our search has a branching factor with upper limit $n$ and a depth factor of upper limit $\left(\begin{array}{l}n \\ 2\end{array}\right)$. Thus we can say this algorithm is bounded above by $\left(\begin{array}{l}n \\ 2\end{array}\right)^{n} \in O\left(n^{2 n}\right)$. There is only constant factor pruning done in this algorithm. Lines 8-9 and 15-16 take time $O(k)$ for every cycle added, which happens $O\left(n^{2} / k\right)$ times. This is completely absorbed into the $O\left(n^{2 n}\right)$ term. 


\subsection{Results and Future Projects}

The two new numbers found with the algorithm developed in this report are $\mathscr{M}(9,4)=1,643,040$ and $\mathscr{M}(9,6)=222,243,840 . \mathscr{M}(11,5)$ was running for 6 weeks and ended early due to a power failure.

The algorithm FIND-CYCLES could be extended and generalized by confining the modification to CALCUlATE-RANGE. If one were to change this method to use the vertex degrees of the vertices in the graph covered by complete cycles and the graph passed in, then FIND-CYCLES could be used to enumerate cycle decompositions of any graph by starting with the complement of the graph in question. Also, if one were to modify CALCUlATE-RANGE in such a way to give a choice set instead of a range, and one were to create a profile for arbitrary graphs, then this algorithm could be used for arbitrary graph decompositions.

In [?], a general algorithm is presented that gives the same labeling for every graph in an automorphism graph. nauty[8](No AUTomorphisms, Yes?) is a C package released by Brendan McKay to find automorphisms of graphs. It was the program [1] used to find $C(n, k), k \neq 3$ in Table 1.1. In fact, because nauty has this canonical form (McKay's Canonical Form, MCF) algorithm built in, one could simply test edges added against the MCF of the graph required and not have to develop their own profile ordering of vertices.

One could obtain better search tree pruning by adding isomorph rejection. This can be done in two simple steps: 1. Adding a global data structure to store the graphs, a modified suffix tree is what I would suggest. 2. folding lines 8-9 and 15-16 on the inside of the for loop, and adding an isomorph against the suffix tree collecting totals of graphs that have nontrivial automorphisms, while ignoring this check if the automorphism group is trivial. Because the $\mathrm{C}$ code used nauty graphs, this is just one call to the algorithm nauty with the correct options set. Adding isomorphism rejection increases computation time at each search node. So if substantial pruning is not achieved then this could result in slower performance. 


\section{Bibliography}

[1] P. Adams and D. E. Bryant, Cyclically generated closed m-trail systems of order $(2 m+$ 1), $m \leq 10$, J. Combin. Math. Combin. Comput. 17 (1995), 3-19. MR 1315637 (96f:05113)

[2] B. Alspach and H. Gavlas, Cycle decompositions of $K_{n}$ and $K_{n}-I$, J. Combin. Theory Ser. B 81 (2001), no. 1, 77-99. MR 1809427 (2002e:05034)

[3] D. Bryant, Cycle decompositions of complete graphs, Surveys in combinatorics 2007, London Math. Soc. Lecture Note Ser., vol. 346, Cambridge Univ. Press, Cambridge, 2007, pp. 67-97. MR 2252790 (2008k:05163)

[4] C. J. Colbourn, Hamiltonian decompositions of complete graphs, Ars Combin. 14 (1982), 261-270.

[5] Bryś K. and Lone Z., A complete solution of the Holyer problem, 4th Twente Workshop on Graph and Combinatorial Optimization (1995).

[6] P. Kaski and P.R. Östergård, The Steiner triple systems of order 19, Math. Comp. 73 (2004), 2075-2092.

[7] D. L. Kreher and T. C. Frenz, An algorithm for enumerating distinct cyclic Steiner systems, J. Combin. Math. Combin. Comput. 11 (1992), 23-32.

[8] B. D. McKay, nauty user's guide (version 2.4), http://cs.anu.edu.au/ bdm/ nauty/nug.pdf.

[9] M. Šajna, Cycle decompositions. III. Complete graphs and fixed length cycles, J. Combin. Des. 10 (2002), no. 1, 27-78. MR 1871681 (2003e:05072)

[10] D. S. Stones, Hamilton cycle decompositions of the complete graph, http://mathoverflow.net/questions/10577/ hamilton-cycle-decompositions, JUN 2011.

[11] M. Tarsi and D. Dor, Graph decomposition if NP-complete: A complete proof of Holyer's conjecture, Siam J. Comput. 26 (1997), 1166-1187. 


\section{Appendix: Source Code}

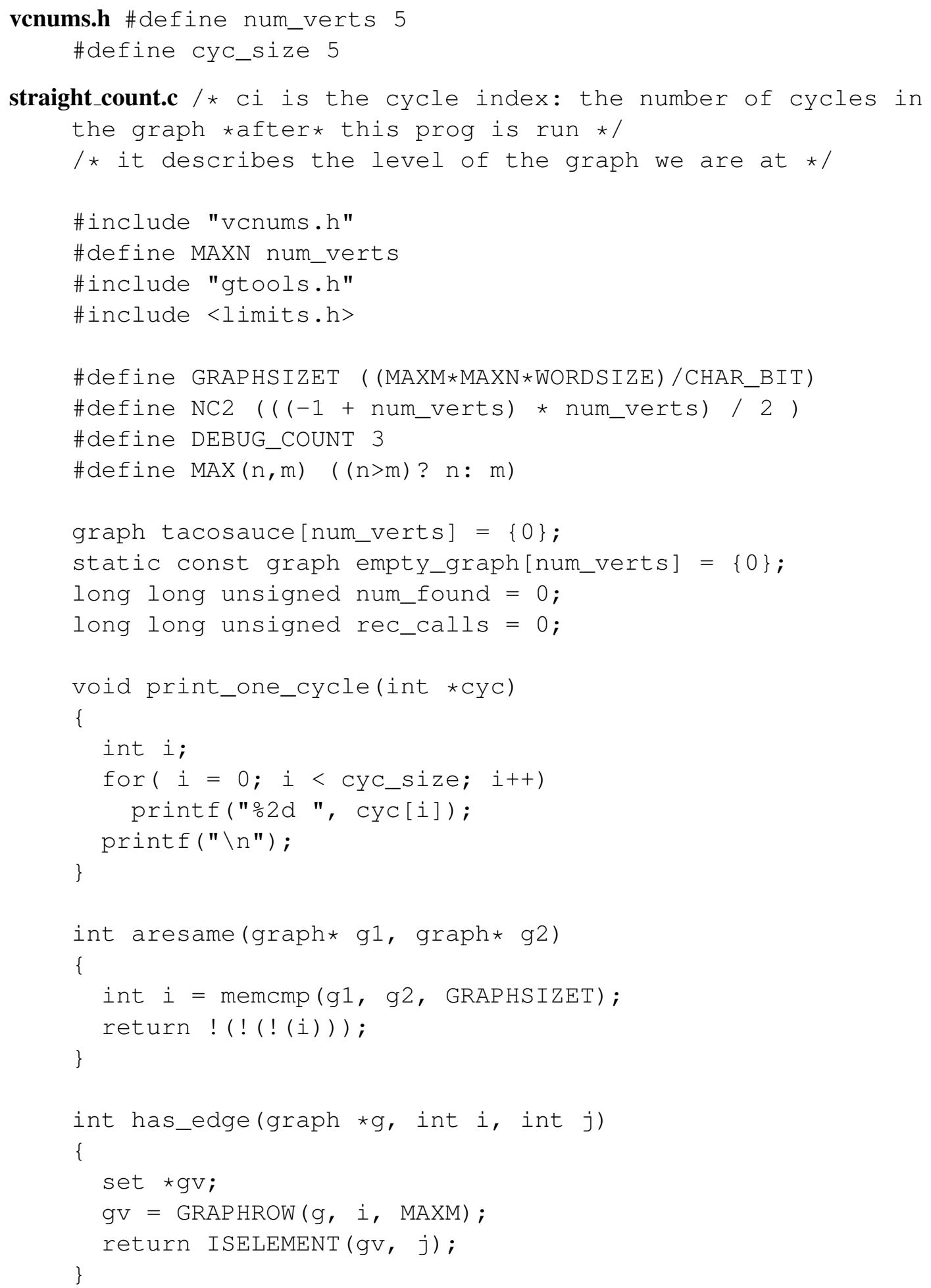




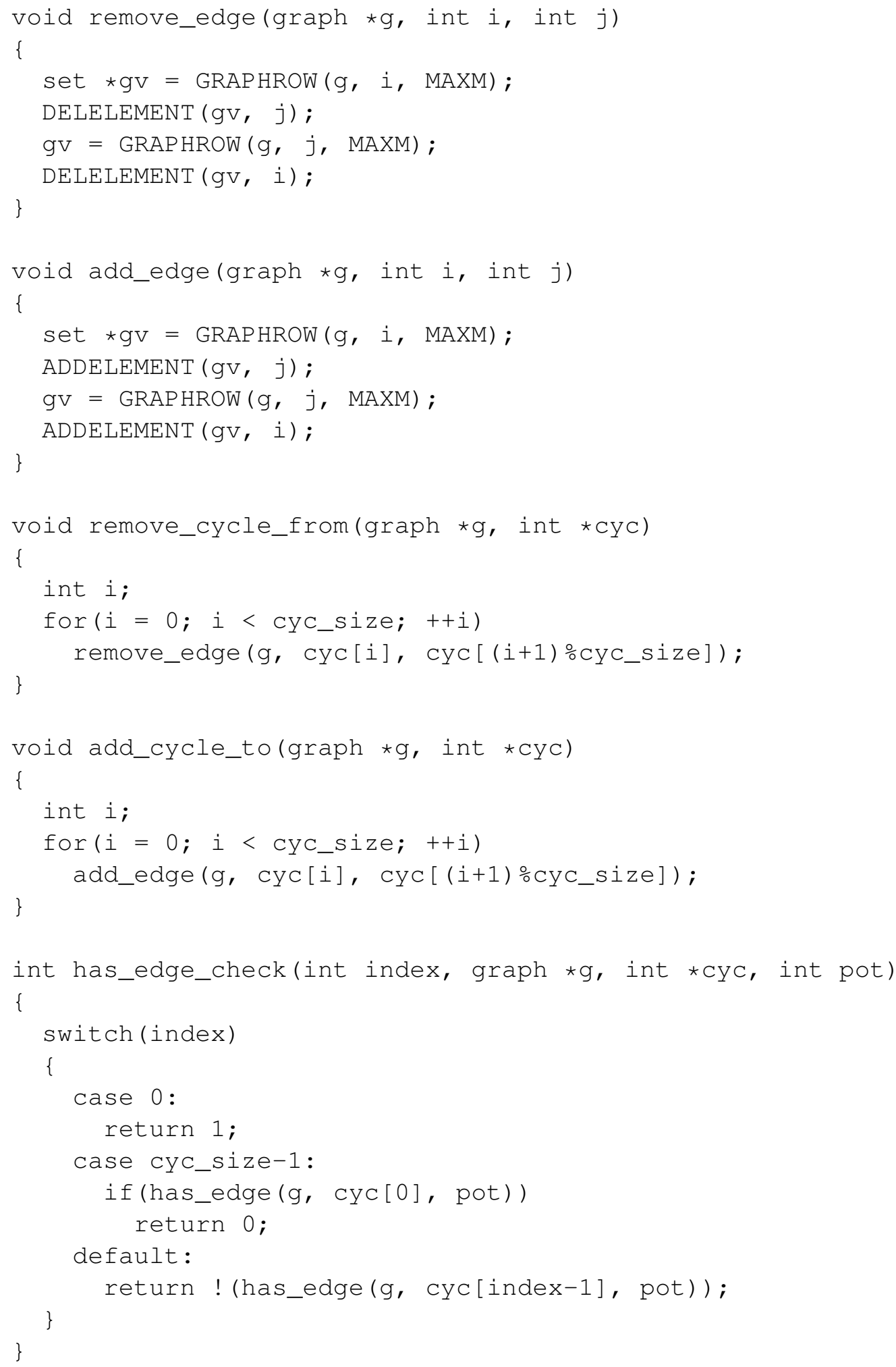




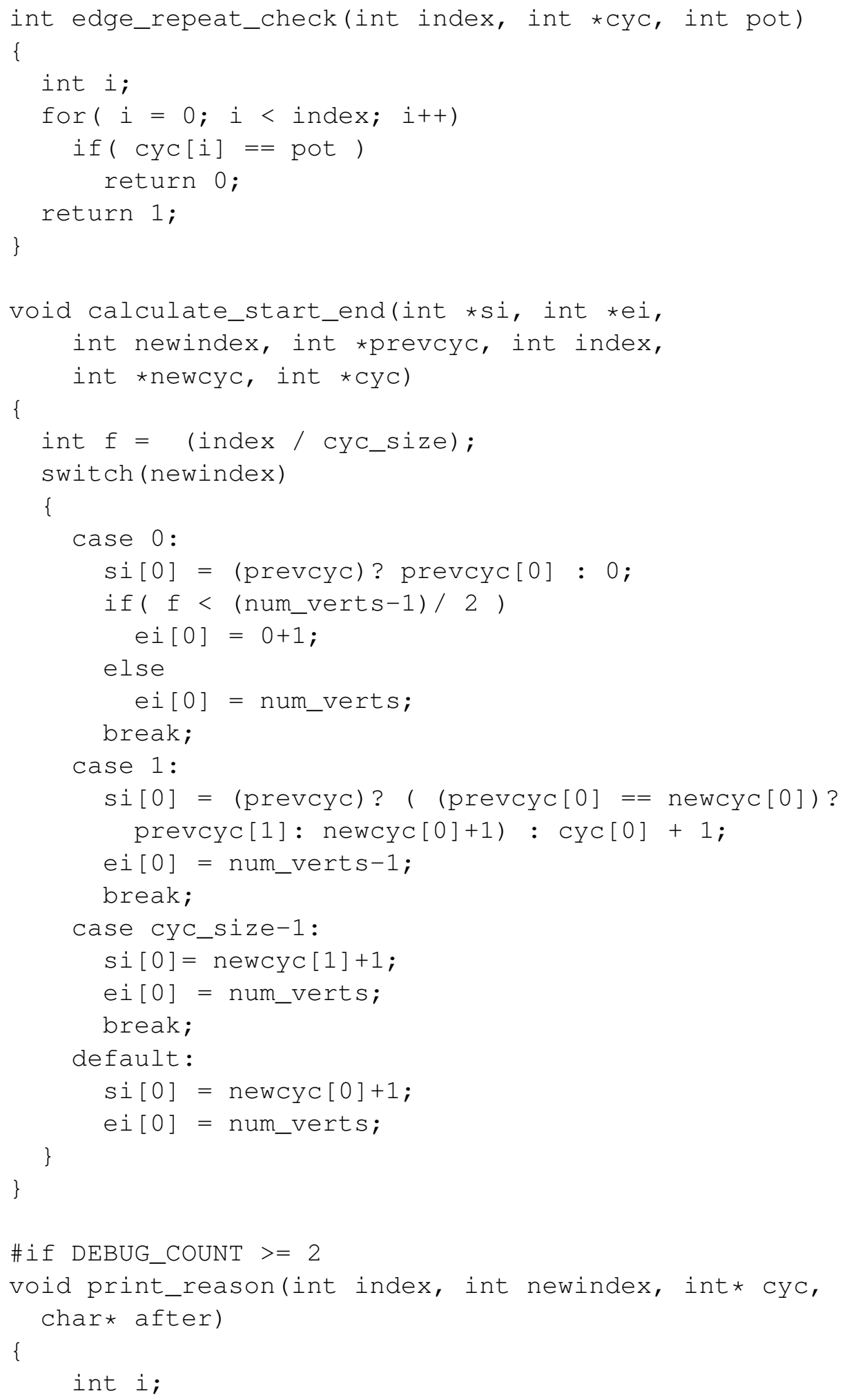




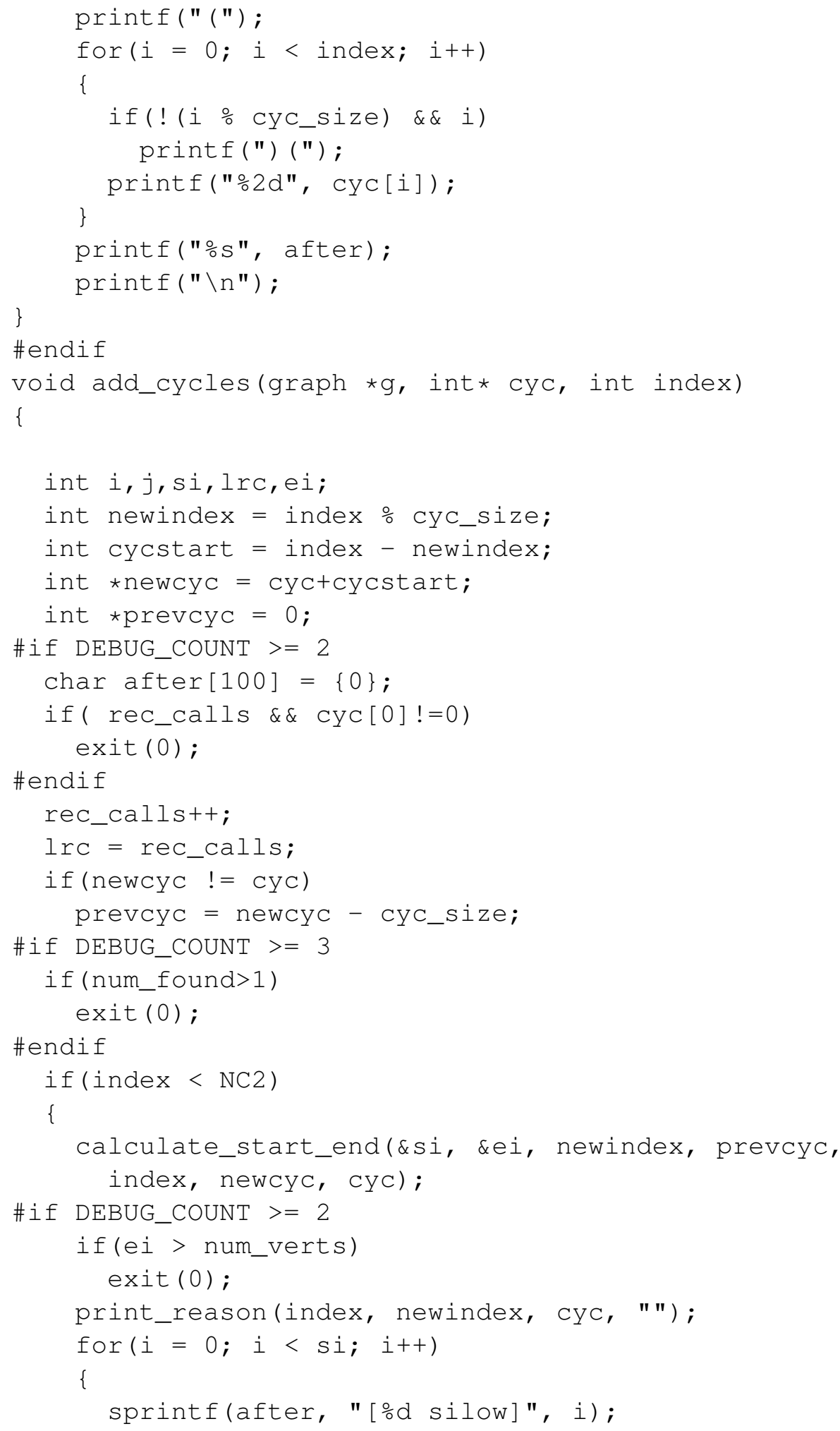




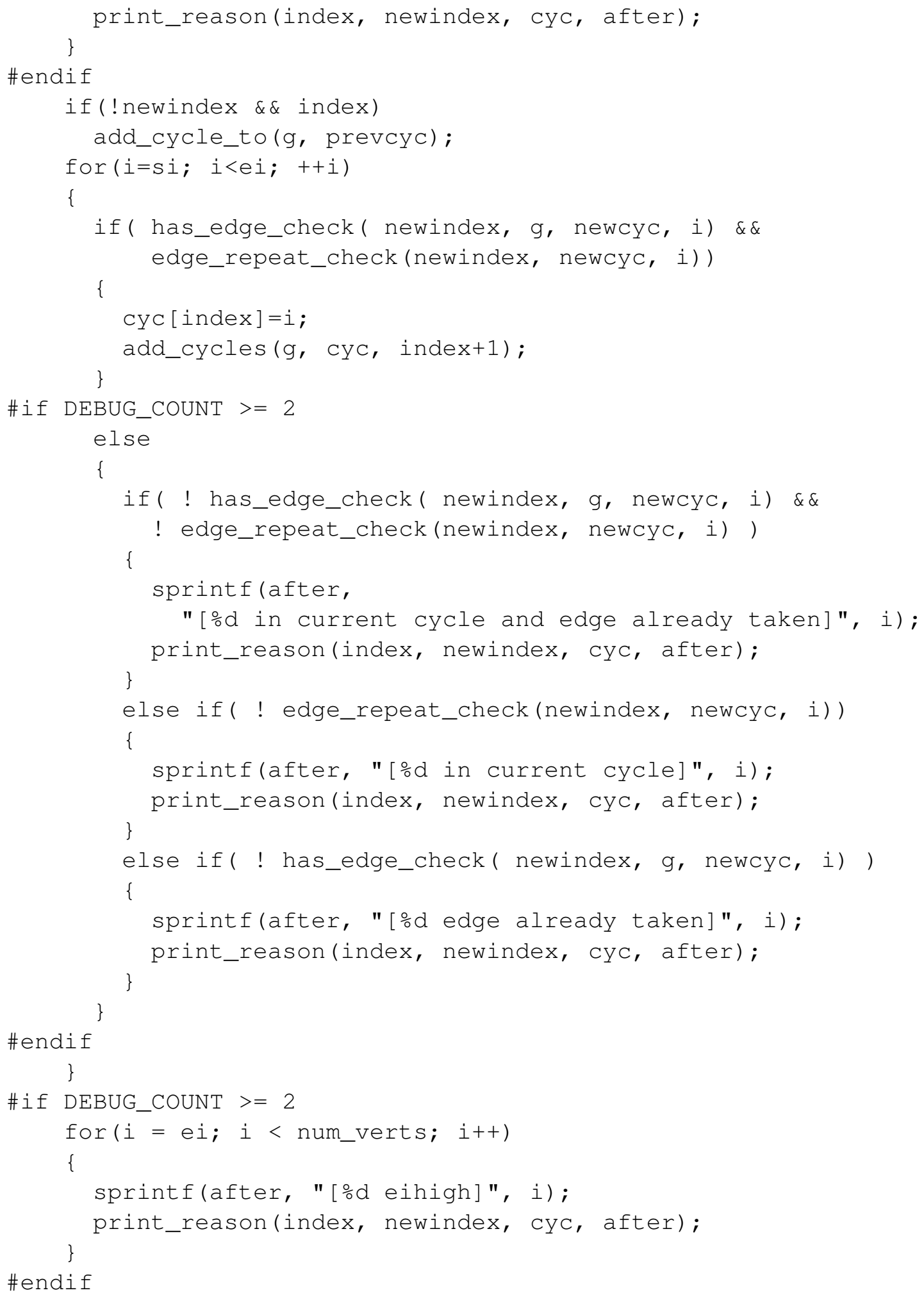




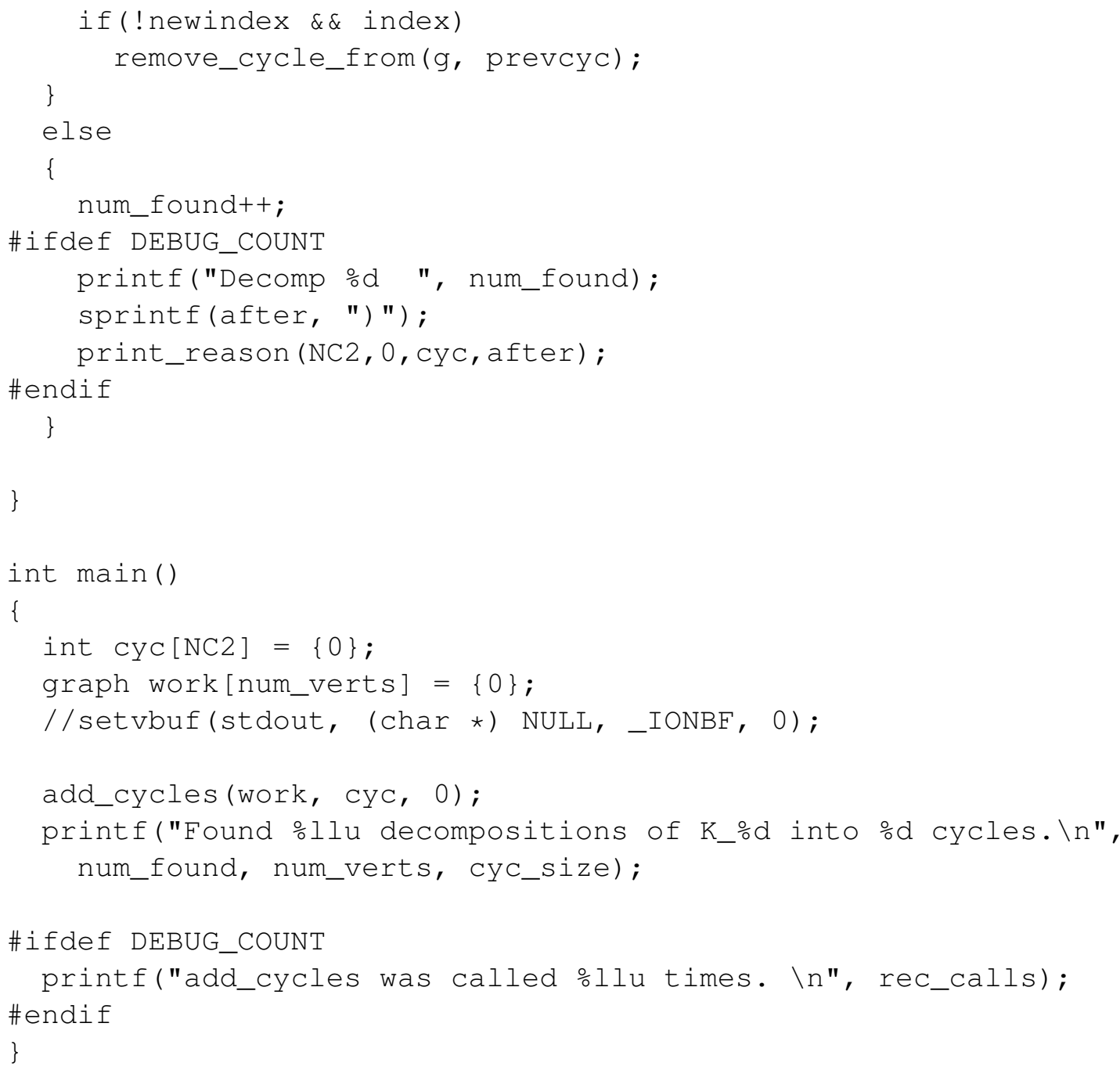

